\title{
Hypothesen und Beobachtungen über die Bedeutung der vertikalen Planktonwanderung.
}

Von

\author{
Dr. G. Burckhardt (Basel). \\ Mit 11 Figuren in Text.
}

"Öber den Grund der Wanderungen wissen wir entschioden nichts Sicheres", schreibt Wesenberg-Lund in seinem 1908 erschienenen Planktonwerk ${ }^{1}$. Ebenda zeigt er, wie überaus nötig es ist, das Verhältnis von Limneticum und Litoral genauer zu erforschen. Ich möchte nun einige hierdurch angeregte Gedankengänge und orientierende Untersuchungen veröffentlichen, die uns vielleicht dem Verständnis der täglichen Wanderung nähern können. Einzelne Ausdrücke, die ich bei Forel, Zacharias und Wesenberg gefunden habe, machen wir den Eindruck, diese Planktologen könnten den Grundgedanken dieser Publikation auch schon gedacht haben; doch ist er meines Wissens noch nicht klar ausgesprochen und bis in seine Konsequenzen verfolgt worden.

\section{Lebt das Plankton auch in der Litoralregion, oder hält es sich fern vom Ufer?}

Schon vor vielen Jahren entschied sich Forel für die zweite, Zacharias für die erste Möglichkeit; letzterer Meinung scheint auch WesenbergLund zuzuneigen. Meine Beobachtungen stimmen, - wenigstens für das größere Zooplankton mit Forels Ansicht überein.

Dafür ein Beispiel: Der Ostarm des Luganersees besitzt am nördlichen Steilufer, nahe der schweizerisch-italienischen Grenze einzelne winzige Alluvionsdelta mit schmalem Schilfsaum. Hier suchte ich dicht aun Pflanzengürtel nach litoral-limnetischen Mittelgliedern. In den Fängen war das

1) Wesenberg-Lund, C., Plankton Investigations of Danish lakes. General Part. 1908 , p. 333. 
Phytoplankton reich vertreten, das Zooplankton dagegen fehlte fast ganz. Und zwar waren die einzelnen Planktonarten nicht in denselben Mengenverhältnissen vertreten, wie zur selben Zeit im Limneticum, sondern abgerundet etwa wie folgende Tabelle zeigt:

$\begin{array}{lcc} & \text { limnetisch } & \text { litoral } \\ \text { Asplanchna priodonta } & 1 & 12 \\ \text { Polyarthra platyptera } & 40 & 200 \\ \text { Anapus ovalis } & 3 & 4 \\ \text { Copepoden-Nauplien } & 20 & 12 \\ \text { Diaphanosoma brachyurum } & 2 & 1 \\ \text { Anuraea cochlearis } & 11 & 4 \\ \text { Notholca longispina } & 13 & 4 \\ \text { Cyclops spec. } & 42 & \left.12^{1}\right) \\ \text { Leptodora hyalina } & 0,1 & - \\ \text { Triarthra longiseta limnetica } & 6 & - \\ \text { Daphnia hyalina } & 10 & - \\ \text { Diaptomus laciniatus } & 31 & -\end{array}$

Polyarthra also überwog im Litoral weit mehr als im Limneticum, außerdem dominierte Asplanchna merkwürdig stark; ebenfalls, doch minder auffallend Anapus; vollständig fehlt die im Limneticum gut vertretene Triarthra. Von den Cladoceren fand ich nur das limnetisch nicht sehr häufige Diaphanosoma.

Unter den Copepoden waren Nauplien besser vertreten als Cyclops, unter diesen vorwiegend die jungen; Diaptomus fehlte ganz.

Am nächsten liegt die Annahme, die Planktozoen seien deshalb am Ufer bei Tage nicht zu finden, weil sie morgens negativ heliotaktisch zur Tiefe sinken. Damit stimmen meine gleichzeitigen Beobachtungen über die tägliche vertikale Wanderung des Luganerseeplanktons sehr wohl überein:

Asplanchna zeigt sehr schwache vertikale Wanderung, ein Viertel aller Organismen bleibt im obersten Meter. Polyarthra weicht zwar etwas weniges zurück, ist aber doch das ausgesprochenste Oberflächenplanktont, bewohnt die obersten $4 \mathrm{~m}$ und hat das Optimum Tags bei $2,3 \mathrm{~m}$. Ähnlich verhält sich Anapus. Anuraea und Notholca ziehen sich bei Tage nicht ganz aus dem obersten Meter zurück, gehen aber in ihrer Hauptmasse in gröBere Tiefen als die bisher genannten. 'Triarthra fehlt der oberen $10 \mathrm{~m}$ Schicht völlig. Unter den Cladoceren wandern die Diaphanosomen am wenigsten tief, immerhin mit einem Tagoptimum bei ca. $14 \mathrm{~m}$. Unter den Copepoden bleiben die Nauplien schon massenhaft im zweiten Meter, junge Cyclops bei $3 \mathrm{~m}$ Tiefe, erwachsene C. strenuus wandern bedeutend tiefer; am allerausgeprägtesten ist die Wanderung bei Diaptomus.

1) Im Litoral nur Junge. 
Aus der Vergleichung ergibt sich: je weiter eine Art zur Tiefe wandert, desto vollständiger fehIt sie bei Tage auch im Litoral. Dies wird manchem Leser selbstverständlich scheinen. A priori wäre aber auch ein anderes Verhalten denkbar: die Tiere wandern wirklich vertikal bis zum Boden und müBten in Untiefen näher der Oberfläche bleiben, als wo ihnen die erwünschte Tiefe zugänglich ist:

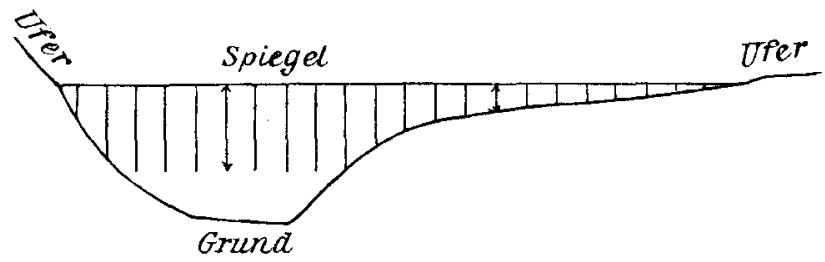

Fig. 1.

Nun sprechen aber meine Beobachtungen und die Forels für die andere Möglichkeit: die negativ heliotaktischen Planktozoen streben unter allen Umständen ihrem Optimum zu; sie sinken, wenn sie am Boden anlangen, schief der Böschung nach weiter:

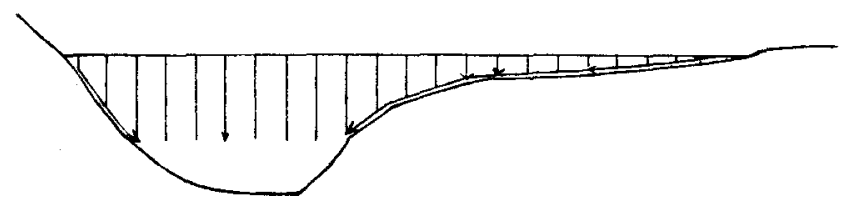

Fig. 2.

Daß Heterocope nicht nur in vertikaler Richtung vor dem Licht flieht, beobachtete Rina Monti am Panalettasee. ${ }^{1}$ )

Wie steigen die Planktozoen Abends wieder auf? Dafür sind zwei Möglichkeiten denkbar:

1. Sie könnten sich ähnlich wie die Gasmolekel verhalten und sich beim Aufsteigen wieder möglichst gleichmäßig in horizontaler Richtung verteilen; die über der Böschung müßten dabei wieder dem Ufer zustreben.

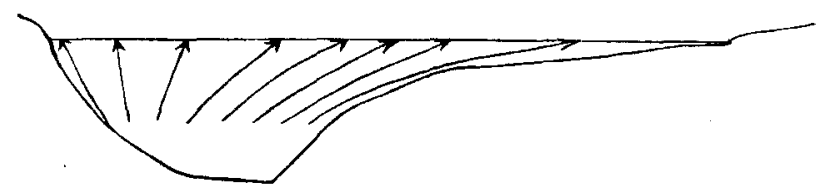

Fig. 3.

1) R. Monti, Physiobiologische Beobachtungen an den Alpenseen zwischen dem Vigezzo- und dem Onsernonetal, Forschungsberichte aus Plön, Teil 12. 1905, p. 82 f. 
2. Oder aber sie steigen auf dem kürzesten Weg dem Dämmerlicht oder besseren Gasverhältnissen entgegen, also senkrecht.

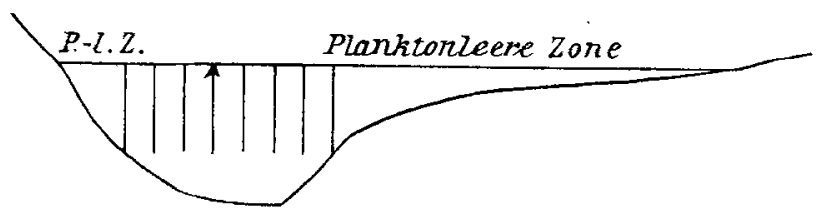

Fig. 4.

Aus diesen zwei denkbaren Steigarten ergeben sich $\mathrm{zw}$ ei verschiedene Verteilungsarten des Oberflächenplanktons bei Nacht; aus der ersten gleichmäBige Verteilung von Ufer zu Ufer, aus der zweiten dagegen eine planktonleere Zone am Ufer und ein planktonreicher Bezirk über der Tiefe.

Zur Annahme senkrechten Aufsteigens muB ich eine Bemerkung vorausschicken: Ich weiB natürlich aus tausendfacher Anschauung, daB die Planktozoen nicht wirklich senkrecht aufsteigen, sondern immer mehr oder weniger schief. Wenn aber die Bewegung eines Tiers durch einen Reiz aufwärts gerichtet ist, und nicht auch durch einen - ja denkbaren - zweiten Reiz seitwärts, so wird es seine vertikale Bewegungskomponente fortwährend beibehalten, die Richtung der horizontalen Bewegung dagegen fortwährend ändern. Es wird im einfachsten Fall eine Schraubenlinie resultieren: am wahrscheinlichsten wird es nach den mannigfaltigsten Schwankungen in allen Richtungen der Windrose schließlich wieder einmal in das Lot über dem Ausgangspunkt kommen. In diesem Sinne glaube ich von vertikalem Aufsteigen sprechen zu dürfen.

Kehren wir zu der durch dieses vertikale Aufsteigen entstehenden planktonleeren Zone zurück. Ihre Breite hängt von zwei Dingen ab:

1. von dem Böschungswinkel:

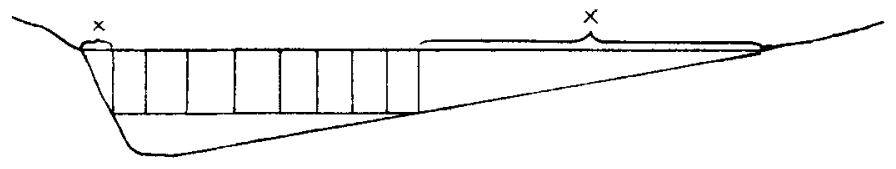

Fig. 5.

2. von der Sinktiefe der Tiere: Planktophyten und nicht wandernde Planktozoen müBten von Ufer zu Ufer gleichmäßig verteilt bleiben, wenig sinkende Rotiferen, Diaphanosomen und Nauplien müßten nur aus einer schmalen Uferzone zurückweichen s. Figur $6 \times$, die bei steiler Böschung leicht ganz verschwinden möchte; und je weiter die übrigen Cladoceren und 
Copepoden täglich zur Tiefe wandern, aus desto breiterer Zone würden sie sich desto vollständiger zurückziehen. Siehe Fig. $6 \times \times$.

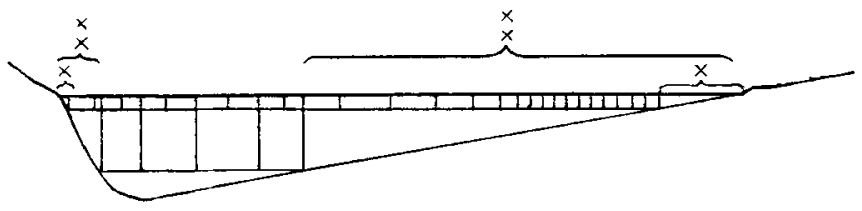

Fig. 6.

Um zu prüfen, ob eine solche der v. W. entsprechende planktonleere Uferzone entstehe, führte ich am 12. Okt. im Silsersee im Oberengadin folgende vertikale Stufenfänge mit einem für Entomostraken geeigneten Griesgazenetz aus:

1. Einen "Totalfang" aus Maximaltiefe. $(71 \mathrm{~m})$

2. Über ca. $50 \mathrm{~m}$ Tiefe 3 Stufenfänge aus $2^{1} / 2$ m, $5 \mathrm{~m}, 10 \mathrm{~m}$ Nachmittags zwischen 3 und 4 Uhr.

3. Utber derselben Tiefe dieselben Stufenfänge Abends um 7 Uhr.

4. Gleich darauf noch vor 8 Uhr nahe dem Ufer Totalfänge vom Boden zur Oberfläche bei $21 / 2 \mathrm{~m}, 5 \mathrm{~m}$ und $10 \mathrm{~m}$ Tiefe.

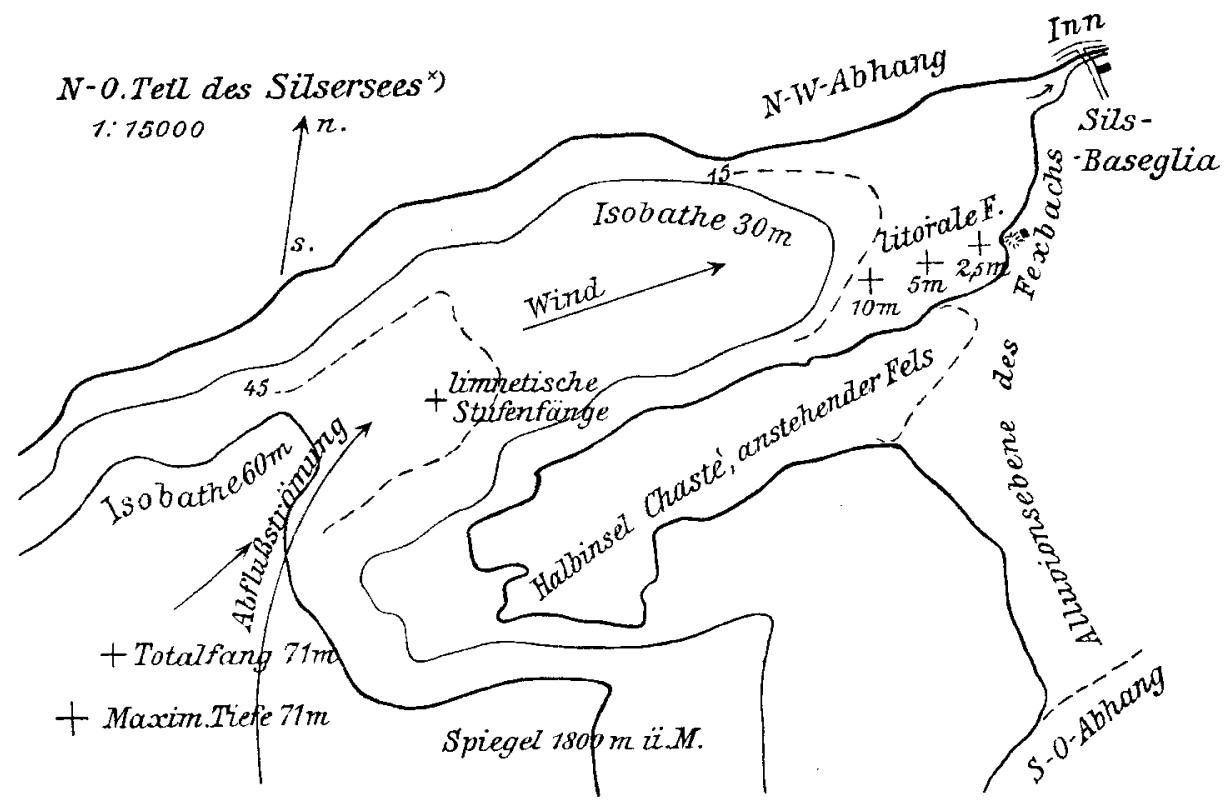

Fig. 7.

*) Beim Lesen des 3. Abschnittes wird man mit mir bedauern, daß verschiedene Umstände mich zwangen nördlich der Chastè-Halbinsel meine Litoralfänge auszuführen statt südlich. Immerhin sind meine Fangstellen wohl noch sehr weit entfernt von 
Die Uferfänge machte ich nach den limnetischen, da zu der Fangzeit noch weiteres Aufsteigen des Planktons zu erwarten war; diese Fehlerquelle wird also die Uferfänge eher zu groß werden lassen. Ich verwertete die Fänge nach der Methode, die ich seinerzeit am Vierwaldstättersee anwandte ${ }^{1}$ ). Zur Publikation ziehe ich die Kategorien zusammen, deren Trennung beim Zählen nicht vollkommen. möglich ist. Die Zahlen des Totalfangs fügte ich bei, um zu zeigen, welche Kategorien der großen Zahlen wegen besonders zuverlässig sind (Bosmina), bei welchen dagegen Zufälle am meisten ausmachen können (Daphnia, Cyclops). Die Stufenfangzahlen setze ich nur als abgerundete Prozentzahlen vom Totalfang in die Tabelle. Die Vergleichung des limnetischen Nachtfangs mit dem limnetischen Tagfang ergibt Angaben über die Intensität der täglichen vertikalen Wanderung, die des limnetischen Nachtfangs mit dem litoralen Nachtfang ermöglicht die Beurteilung der "Flucht vor dem Ufer". Für diese beiden berechne ich (aus den rohen Fangzahlen, nicht aus den in der Tabelle enthaltenen Prozentzahlen) je einen Quotienten:

limn. Nachtfang: litor. Nachtfang = Uferfluchtquotient.

limn. Nachtfang: limn. Tagfang $=$ Wanderungsquotient.

Würden die Planktonten sich beim Aufsteigen sofort gleichmäßig verteilen, entstünde also keine planktonarme Uferzone, so fänden wir den Uferfluchtquotienten $=1$; würden die Planktonten über der Böschung nach der allerersten Annahme gar nicht schief sinken, so dürften wir unter Annahme gleicher Lebensbedingungen sogar annähernd gleiches Totalquantum auf der Böschung und über der Tiefe erwarten und erhielten durch Division eines Teils des limnetischen Planktons durch das gesamte Litoralplankton einen Uferfluchtquotienten $<1$. Tatsächlich ist er für alle Kategorien beträchtlich $>1$, und zwar ist er - geringfügige Ausnahmen abgerechnet - für die erste Stufe $>$ als für die zweite, für diese $>$ als für die dritte. Damit ist eine deutliche Uferflucht festgestellt.

Mit Berücksichtigung des geringen Zeitunterschiedes zwischen den

Wolterecks Zone, wo die AusfluBströmung sich bemerkbar zu machen beginnt. Ist doch der See an der Fangstelle noch kaum weniger breit als am Eingang des N-OTeiles und noch gewaltig breit und sehr tief im Vergleich zum AusfluB, einem recht unbedeutenden Bach (trotz dem Namen Inn). Ich schätze den Querschnitt auf das 600 bis 3000 fache des Ausflusses; dem entspricht das Verhältnis der Abflußgeschwindigkeit. Zur Fangzeit war der Spiegel des Sees tief, der AbfluB also relativ schwach.

Die S-0-Bucht ist übrigens auch nicht ganz einwandfrei: in ihren äußeren Teil, vielleicht auch in ihre Mitte mündet der weitaus bedeutendste Zufluß des Sees; dazu dürfte am N-O-Ufer dieser Bucht ein möglicherweise vorhandener Grundwasserzufluß vom Fexbach her ganz bedeutend mehr ausmachen als an meinen Fangstellen.

1) S. meine Quantitativen Studien über das Zooplankton des Vierwaldstättersees. Mitt. naturf. Ges. Luzern 1900. p. 83 ff.

Revue d. ges. Hydrobiol. u. Hydrogr. Bd.III. H. 1 u. 2. 


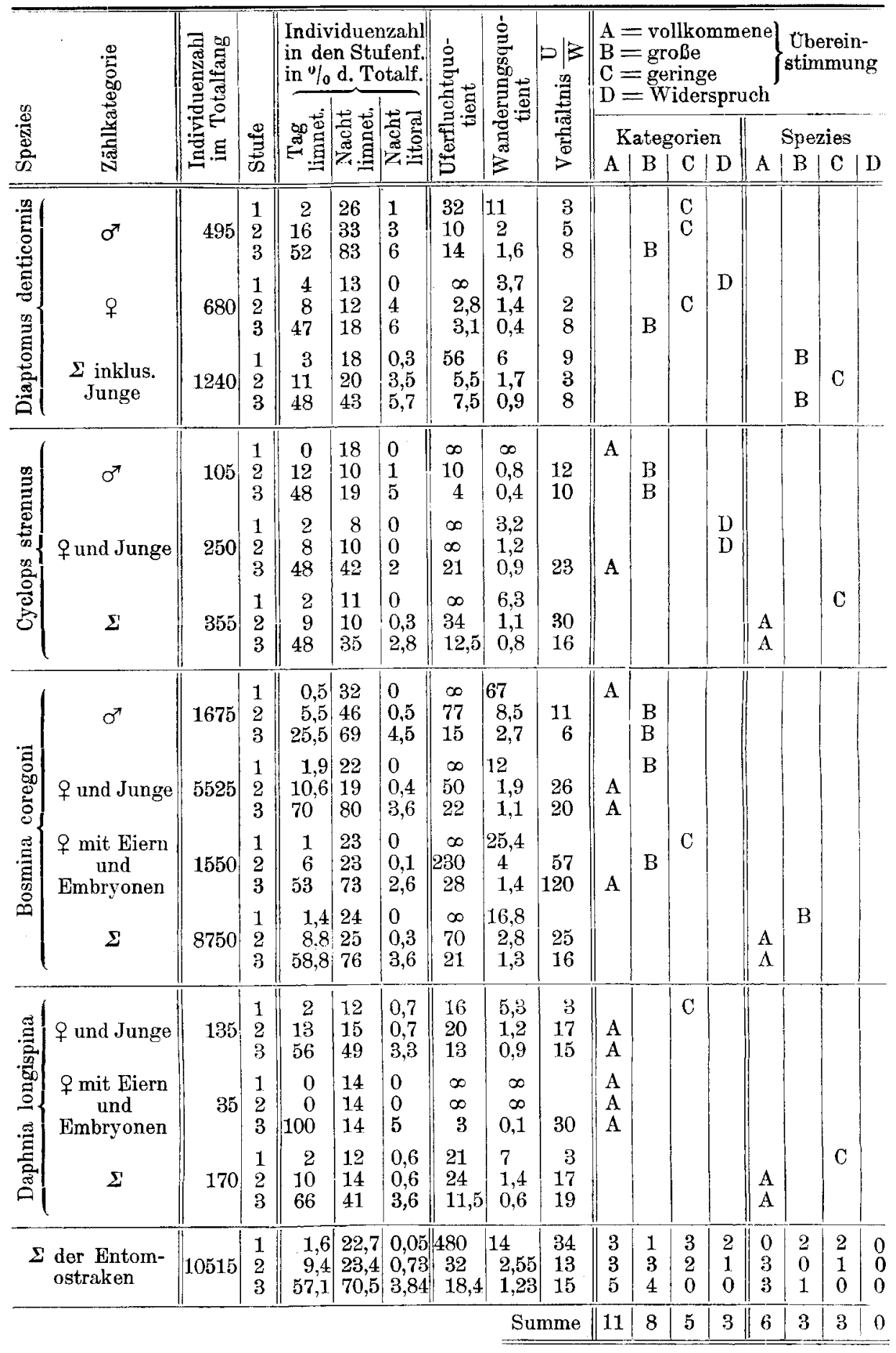


limnetischen und den litoralen Fängen ergibt sich: um 8 Uhr Abends fand sich in den entsprechenden Stufen am Ufer nur

$$
\begin{array}{llll}
\text { der } & 500 . & (\text { bei } & 2^{1 / 2} \mathrm{~m}-0 \mathrm{~m} \text { ) } \\
\text { der } & 35 . & \text { (bei } 5 & \mathrm{~m}-0 \mathrm{~m} \text { ) } \\
\text { der } & 20 . & \text { (bei } 10 & \mathrm{~m}-0 \mathrm{~m} \text { ) Teil von derim Lim- }
\end{array}
$$

netikum erbeuteten Entomostrakenmenge. Von der Gesamtmenge (vom Boden bis zur Oberfläche) habe ich am Ufer nur den

$$
\begin{aligned}
& \text { 2000. }\left(2^{1} / 2 \mathrm{~m}-0 \mathrm{~m}\right) \\
& \text { 137. }(5 \mathrm{~m}-0 \mathrm{~m}) \\
& \text { 26. }(10 \mathrm{~m}-0 \mathrm{~m}) \text { Teil gefunden. }
\end{aligned}
$$

Ist diese Uferflucht eine Folge der v. W., so muß sie bei den besonders deutlich wandernden Planktozoen ebenfalls besonders deutlich auftreten. Das $m u B$ sich aus der Vergleichung der Uferfluchtquotienten und Wanderungsquotienten ergeben. Den Wert des letzteren müssen wir aber vorerst noch kritisieren. Je allgemeiner die Angehörigen einer Spezies am Tag unter $\mathbf{2}^{1 / 2} \mathrm{~m}$ sinken und je allgemeiner sie Nachts darüber steigen $^{1}$ ), desto gröBer wird sich der Wanderungsquotient ergeben. Die Uferflucht hängt nun nach meinen Darlegungen nur mit der Frage zusammen, ob die betreffende Spezies recht vollständig unter die Stufe sinke, nicht damit, ob sie Nachts eifrig an die Oberfläche andränge, also nur mit dem einen der beiden Komponenten des Wanderungsquotienten. Doch wird durch das starke Andrängen zur Oberfläche nicht nur der Wanderungsquotient vergröBert, sondern in demselben MaBe auch der der Uferflucht. Die beiden Zahlen werden also zur Prüfung meiner Hypothese brauchbar sein.

Dagegen wird dem Leser nicht entgehen, wie summarisch meine $\mathrm{Be}-$ obachtung der v. W. war. Hätte ich sie wirklich so kennen lernen wollen, daß ich ihre Wirkung auf die Uferflucht bemessen könnte, so hätte ich für jede Kategorie das Maximum der Tiefwanderung konstatieren müssen. Diese bätte wohl früher am Tage stattgefunden und hauptsächlich nicht für jede Kategorie gleichzeitig. Für eine so genaue Untersuchung fehlt mir die Zeit. Sie hätte wohl auch nicht genügt; denn es ist nicht anzunehmen, daß nur die größte Tiefe, die an dem einen Tag erreicht wurde, in Betracht käme. Wir haben ja noch keine Ahnung, wie schnell die durch $v$. W. erreichte Uferflucht durch Strömungen und Lokomotion der Planktonten wieder verwischt wird. Das wird erst durch hundertfache Erfahrung zu ergründen sein. Meine Beobachtung ergibt also nur einen Bruchteil der in Betracht fallenden v. W., und, - was das mißlichste ist, - für jede Kategorie einen andern Teil.

1) Darauf, daß in der Regel nicht alle Individuen einer Art, eines Sexus, eines Niters, in ihrer Gesamtheit wandern, hat Hofer zuerst aufmerksam gemacht (1899, Bodensee). 


\section{$-164-$}

Wären diese Unvollkommenheiten nicht da, und könnten wir auch die bekannten Fang- und Zählfehler vermeiden, so hätten wir erwarten müssen, daß jedesmal der Wanderungsquotient und der Uferfluchtquotient gleich groß geworden wäre. Unter diesen Umständen aber erwarten wir einen kleinern Wanderungsquotient, wie er sich ausnahmlos ergeben hat. Wir verlangen auch, daß die beiden Reihen im Ganzen ungefähr parallel laufen. Dies ergibt sich auch recht deutlich:

Kategorien mit besonders ausgeprägter $\mathrm{v}$. W. zeigen auch besonders starken Uferfluchtquotient:

$$
\begin{gathered}
\text { Bosmina- } \sigma^{7} \\
\Rightarrow \quad-E i e r-9 \\
\text { Daphnia-Eier- }+9
\end{gathered}
$$

Kategorien mit mittlerer v. W. zeigen auch mittelstarke Uferflucht:

$$
\begin{aligned}
& \text { Diaptomus }-\sigma^{x} \\
& \text { Cyclops }-\sigma^{T}
\end{aligned}
$$

Geringer v. W. entspricht auch geringe Uferflucht bei

$$
\text { jungen Daphnien und }
$$

Diaptomus- +9

Einen auffallenden Widerspruch zeigt die Kategorie Cyclops $q$ und Junge, doch nur in den ersten zwei Stufen zunächst dem Ufer, wo die möglichen Fehler der kleinen Zahlen wegen am größten sind. Am wenigsten Ubereinstimmung zeigen im Allgemeinen

1. die Kategorien mit kleinen Zahlen (Bosmina im Gegenteil dio größte bei besonders großen Quantitäten),

2. die erste Stufe, wo besonders der Litoralfang so winzig war, daß die Zahlen für die einzelnen Kategorien eigentlich gar nicht ernst zu nehmen sind. ${ }^{1}$ )

Ich darf also meine am Anfang gestellte Frage nach dieser orientierenden Untersuchung etwa so beantworten: es existiert Tag und Nacht am Ufer eine zooplanktonleere Zone, deren Breite für die einzelnen Arten verschieden ist und sich nach ihrer Wanderungstiefe richtet. Die planktonleere Zone entsteht durch die vertikale Wanderung.

Dagegen existieren mehrere Faktoren, welche die Uferzone wieder mit Plankton bevölkern können:

1) Dieser Fang enthielt außerdem massenhaft Hydra fusca vom Grunde her, weshalb leicht Planktontiere beim Zählen können übersehen worden sein; habe ich 7 Stück übersehen, so resultiert fūr die Gesamtzahl nicht das Verhältnis $\frac{U}{\bar{W}^{r}}=34$, sondern $=15$ wie bei 2. oder 3. Stufe. 
1. Die aktive Bewegung der Planktozoen wird, da sie horizontal nicht gerichtet ist, nur sehr wenig ausmachen können.

2. Sehr bedeutend ist unter Umständen die passive Bewegung des Planktons durch die vom Wind erzeugte Strömung des Oberflächenwassers. Hingegen scheint auch dadurch die leere Zone nicht schnell zu verschwinden, herrschte doch während der meinen Beobachtungen im Silsersee vorhergehenden Tage und am selben Tag bis Sonnenuntergang starker S-W, der das Oberflächenplankton gerade in die Bucht, wo ich die Uferfänge ausführte, bringen mußte.

3. Die Abflußströmung des Sees nähert in den sich gegen den Ausfluß zu verjüngenden Seeteilen das Wasser dem Ufer. Darauf werde ich später zurückkommen.

Wenn wir uns diese tägliche Periode der Entfernung vom Ufer durch das Niedersinken am Morgen und der Annäherung ans Ufer durch die drei eben genannten Faktoren im übrigen Teil des Tages vorstellen, so bemerken wir, daß in der Grenzzone (zwischen der armen Uferzone und dem eigentlichen Limneticum) eine besonders planktonreiche Region entstehen kann, wenn nicht die sie verwischenden Faktoren noch schneller wirken als die sie bildenden. Diese Zone der fortwährenden Anreicherung ist frei-

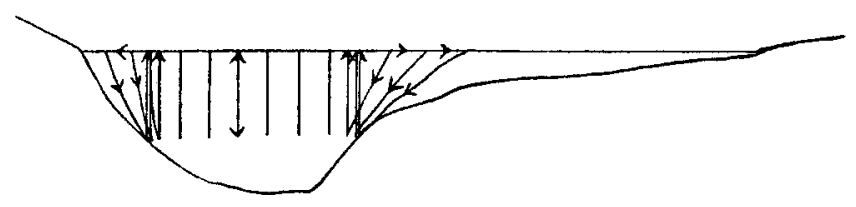

Fig. 8.

lich nicht sehr leicht zu konstatieren, da sie für jede Art und für jedes Altersstadium wieder eine andere ist, je nach der Tiefe der v. W. Es wird sehr genauer Methoden bedürfen (Pumpfänge vom Boden bis zur Oberfläche), um sie einwandfrei zu beobachten. Doch will ich bemerken, daß ich in der betreffenden Zone des Vierwaldstättersees und des Luganersees gelegentlich Schwankungen der Individuenzahl von etwa $20 \%$ gefunden habe, die so zu deuten sein könnten.

\section{Der Selektionswert der die planktonleere Zone bedingenden tägliehen vertikalen Wanderung.}

Es ist klar, daß die Entfernung des Planktons aus der Litoralregion und also die sie bedingende tägliche vertikale Wanderung böchst „zweckmäBig" sind. Die tiefwandernden Planktonten haben für die Erhaltung der Individuen ganz bedeutendes vor den nicht wandernden voraus. Denn am Ufer drohen den Planktozoen die mannigfaltigsten Gefahren: 
1. die Brandung;

2. größere Temperaturschwankungen;

3. Wasser aus Zuflüssen, das bei uns meist ärmer an Nahrung und, wenn es nicht aus nahen Quellen stammt, weniger gut temperiert ist;

4. Planktophagen;

5. der AbfluB, der von ganz passiv bewegtem, gleichmäßig verteiltem Plankton fortwährend große Mengen aus dem See und damit auch oft aus den allein genügenden Lebensbedingungen wegführt.

Es liegt mir daran, deutlich zu sagen, welche von den Faktoren, die die tägliche v. W. bedingen, durch meine Annahme erklärt werden können, und für welche andere Gründe bestehen bleiben oder noch gesucht werden müssen.

Die tägliche vertikale Wanderung beruht auf einer jeder Spezies und jedem Altersstadium eigentümlichen Regulation, also auf der Organisation.

Der die v. W. regulierende Reiz ist das Licht, sicher beim Niedersinken, nach einigen Forschern ${ }^{1}$ ) auch beim Aufsteigen, und zwar erweisen sich die Planktozoen als auf geringe Lichtintensität abgestimmt.

Warum müssen nun die Planktozoen Dämmerungstiere sein? Könnten sie nicht alle, - so gut wie viele andere, sogar einzelne von ihnen selbst, - sich an den großen täglichen Lichtwechsel angepaßt haben? Diese Frage teilt sich in zwei, die nach dem Grund des Niedersinkens, und die nach demjenigen des Aufsteigens.

Nehmen wir die zweite vorweg: die Frage nach der Erhaltungsmäßigkeit des Aufsteigens zur Oberfläche, die Frage, warum nicht alle Planktontiere in der Region bleiben, wo die Nacht nur mit der Dämmerung wechselt, wie es bei der limnetischen Triarthra der Fall zu sein scheint. Eine Antwort darauf versuchte ich $\left.1900^{2}\right)$ zu geben, indem ich die Gasverhältnisse zur Erklärung der v. W. heranzog, die schon Birge zum Verständnis der gleichbleibenden vertikalen Verteilung benutzt hatte. $\left.{ }^{3}\right)$

Der $\mathrm{CO}_{2}$-Gehalt des Wassers nimmt von der Tiefe gegen die Oberfläche hin $a b$, in dieser Beziehung werden die Lebensbedingungen bis zur Oberfläche immer besser. Dies kann das Aufsteigen und auch das Andrängen zur Oberfläche erklären. (Die Verteilung der planktophytisehen Nahrung dagegen kann nicht den Ausschlag geben, denn diese ist gar nicht immer so überwiegend an der Oberfläche lokalisiert, wie es gewisse

1) Für Bosmina coregoni und Hyalodaphnia hat Ruttner dies nachgewiesen. Forschungsberichte aus Plön, Teil 12. p. $48 \mathrm{f}$.

2) siehe meine quantitativen Studien, p. $255 \mathrm{f}$.

$\left.{ }^{3}\right)$ Birge, Plankton Studies on Lake Mendota Il. Transact. Wisconsin Acad. Sc, v. X. p. $423 \mathrm{ff}$. 
Planktozoen Nachts sind.) Die Annahme ist später durch Loebs ${ }^{1}$ ) Versuche bestätigt worden.

Aber gerade diese besseren Bedingungen, die wir an der Oberfläche kennen lernen, vergrößern das Bedürfnis nach einem zwingenden Grund für das Niedersinken. A priori müßte man doch annehmen, diese besseren Bedingungen hätten Formen heranzüchten können, die sich an die wechselnde Lichtintensität anpassen und also Tag und Nacht in der Schicht des besten Gasgehaltes bleiben.

Diesen Teil der Frage nach den Gründen der vertikalen Wanderung suche ich nun mit meiner Hypothese zu beantworten, d. h. von diesem periodischen Niedersinken aus Schichten mit günstigen Gasbedingungen in solche mit schlechtern, möchte ich beweisen, daß es erhaltungsgemäB ist, daB es Selektionswert hat.

Seine Erhaltungsgemäßheit liegt darin, $d a ß$ es die Tiere von dem gefährlichen Ufer wegführt und zwar immer von neuem, wenn sie durch irgend welche Vorgänge (durch Wind- und Abflußströmung) gegen das Ufer hin getrieben worden sind.

Diese ErhaltungsmäBigkeit erlaubt uns anzunehmen, daß diø Abstimmung auf Dämmerlicht oder also die tägliche vertikale Wanderung durch die natürliche Auslese herangezüchtet oder wenigstens erhalten worden sei. (Auf diese zweite Möglichkeit werde ich am Schluß zu sprechen kommen).

\section{Die Flucht vor dem Ausfiuß.}

Woltereck ${ }^{2}$ ) teilte 1908 eine interessante Beobachtung aus den Lunzer Seen mit, die ihm aus anderen Gegenden bestätigt wurde: Der AusfluB entführt massenhaft Phytoplankton, dagegen fast kein Zooplankton; die Cladoceren und Copepoden scheinen nie mitgerissen zu werden; weniger strikte gilt dies für die Rotiferen. Woltereck diskutiert kurz die verschiedenen denkbaren Erklärungsversuche: negativen Rheotropismus und optische Wahrnehmung schließt er aus; er hält dagegen für möglich, daß Gleichgewichtsänderungen eine Rolle spielen, ähnlich wie beim Niedersinken des Planktons in Folge von Wellengang. Er betont aber, daB die Tatsache noch als völlig dunkel gelten müsse.

Es wäre nun möglich, daß sich diese AusfluBflucht nur als ein Spezialfall der Uferflucht herausstellte. Wenn die vertikal wandernden Planktonten der seichten Uferzone fehlen, so fehlen sie auch dem Ausfluß. In der Gegend des Ausflusses entfernen sich die Vertikalwanderer jeden Morgen leicht weiter vom Ausflub, als sie ihm während 24 Stunden

1) Siehe Lit. in meinem Ref. über Loeb und Bauer, diese Zeitschr., Bd. III, S. 196.

2) Notiz über Plankton und Seenausfluß. Diese Zeitschrift, Bd. I. p. 303 f. 
durch den Durchflußstrom genähert werden, und retten sich so ihrer Kolonie.

Diese Lebensrettung mag ja wohl ein Hauptgrund, vielleicht sogar der wichtigste, für den Zuchtwert der v. W. sein.

Bekanntermaßen gibt es einzelne planktonarme oder planktonleere Gebirgsseen in tiefern Lagen. Ihre Planktonarmut könnte in einem für die Flucht vor dem Ausfluß ungünstigen Bodenprofil begründet sein. Als Bei-

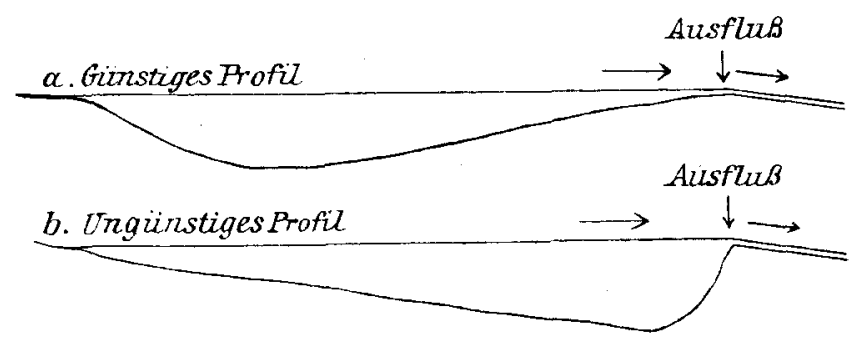

Fig 9: a, und b.

spiel dürfte der Klöntalersee gelten können, der noch $200 \mathrm{~m}$ vom Ausfluß $20 \mathrm{~m}$ tief ist und dessen Niveauschwankungen seine Wassermenge im Verhältnis von 3:5 ändern lassen. Dieser See zeichnet sich durch große Armut an Plankton aus, besonders im Frühling zur Zeit des höchsten Wasserstandes, und hat außerdem in den letzten Jahrzehnten seinen limnetischen Diaptomus ganz und seine Daphnia beinahe verloren. ${ }^{1}$ )

Mit der Annahme eines Zusammenhangs zwischen Ausflubflucht und v. W. stimmt überein, daß nach Wolterecks Beobachtung die im Allgemeinen am weitesten vertikal wandernden Entomostraken dem AusfluB sozusagen ganz entgehen, die nur zum Teil vertikal wandernden Rotiferen nur zum Teil, die nicht aktiv wandernden Algen gar nicht.

So wäre unerwartet schnell die dunkle Frage der Ausflußflucht erhellt.

\section{Lokale Verschiedenheit der vertikalen Wanderung.}

In verschiedenen Seengebieten scheinen nah verwandte Planktozoen oder gar Vertreter derselben Art sich sehr verschieden zu verhalten. Während in den Seen des Alpenrandes und der Alpen die vertikale Wanderung ein höchst auffälliger Vorgang ist, entging er im baltischen Seengebiet lange der Beobachtung ${ }^{2}$ ) und ist in verschiedenen andern Seenfluren noch

1) siehe Heuscher, Untersuchungen über die biologischen und Fischereiverhältnisse des Klöntalersees. 1903, Pfäffikon-Zürich.

9) Immerhin fehlt Diaptomus im Plönersee tags in den obern $3 \mathrm{~m}$ fast gānzlich und ist zahlreich erst unter $5 \mathrm{~m}$. 
gar nicht beobachtet worden. Daraus auf vollständiges Fehlen zu schließen, wäre aber nach unseren bisherigen Erfahrungen nicht berechtigt.

Die nächstliegende Erklärung war bisher die verschiedene Transparenz: doch konnte dies nicht wohl befriedigen, da gerade der wichtigste Trübungsfaktor der Alpenrandseen, der feine Gletscherdetritus in den baltischen Seen gänzlich fehlt.

Nun führt meine Hypothese auf eine andere Erklärung: Wir dürfen annehmen, daB die Züchtung der vertikalen Wanderung entweder noch heute andauert, oder daß bei Fehlen des züchtenden Moments die in diesem Fall unnötige Arbeit des Vertikal-Wanderns wieder verloren geht oder weggezüchtet wird.

In einem flachen See mit sehr wenig geneigten Böschungen wird geringe vertikale Wanderung die Planktozoen in große Uferferne bringen (siehe Fig. 10a).

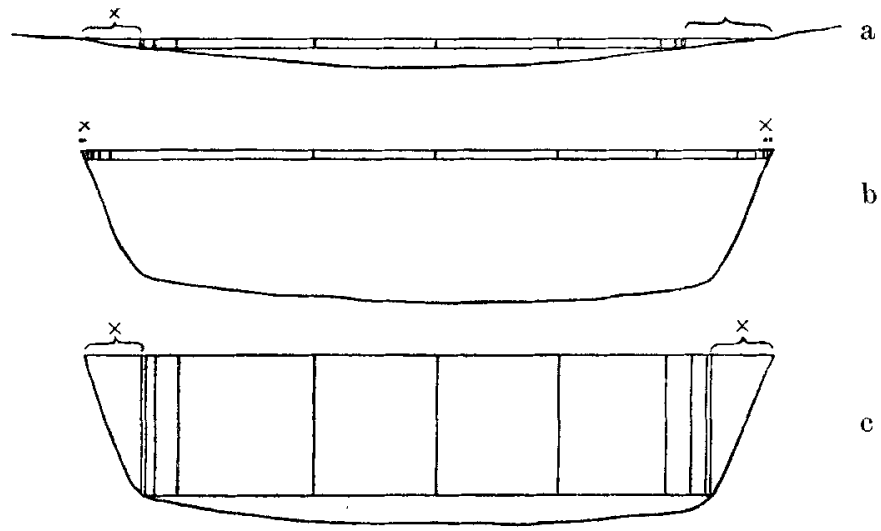

Fig. 10: a., b. und c.

In einem See dagegen mit steilen Böschungen wird dieselbe vertikale Wanderung nur eine schmale planktonarme Zone zur Folge haben (b), oder zur Erreichung derselben Uferflucht wie beim ersten See wird eine weitere vertikale Wanderung nötig sein (c).

(An ganz vertikalen Böschungen wäre die vertikale Wanderung allen Erfolges bar; doch kommen solche ja nicht in praktisch bedeutender Ausdehnung vor.)

Daraus folgt, daß in Seen mit steiler Böschung eine viel bedeutendere vertikale Wanderung gezüchtet werden muB, als in solchen mit sanften Abhängen.

In der Tat zeichnen sich die Seen des Alpenrandes und der Alpen vor den meisten anderen europäischen Seen durch große Steilheit der Gehänge aus. Diese ist überhaupt ein Charakteristikum der Hochgebirgsseen: sie wächst mit dem Korn des an den Ufern abgelagerten Alluviums; dieses 
Korn des Alluviums hängt seinerseits vom Gefälle der Zuflüsse ab. Also je steileres Gebirge im Zuflußgebiet, desto gröberes Alluvium, desto steilere Hänge, desto weiter in die Tiefe muB sich die vertikale Planktonwanderung erstrecken, und umgekehrt. Es müßten, wenn unsere Folgerungen ganz richtig sind und nicht andere Verhältnisse noch bestimmender auf die vertikale Wanderung einwirken, Gebirgsseen weit wanderndes, Flachlandseen schwach wanderndes Zooplankton beherbergen. Dies müBte durch weitere Beobachtung bestätigt werden. ${ }^{1}$ )

In Seen mit sanfter Böschung werden auch leicht sekundäre Depressionen am Abhang auftreten. Diese bewirken eine bedeutende Komplikation in der Wirkung der vertikalen Wanderung und heben unter Umständen ihre "Zweckmäßigkeit" bis zu einem gewissen Grade auf. Die mutmaßlichen Folgen davon sind entweder Wegzüchtung der vertikalen Wanderung (resp. Verkleinerung derselben, bis sie nicht mehr bis zur Tiefe der Barren reicht) oder aber - wenn die vertikale Wanderung noch vorhanden ist, - ungleichmäBige horizontale Verteilung in der Art, wie die beigegebene Figur zeigt.

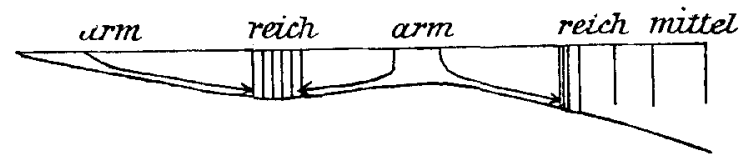

Fig. 11.

AuBer dem Böschungswinkel könnte hier auch noch in Betracht fallen, daß nicht in allen Seen und Seengebieten die Ufer gleich gefährlich sind. So wäre auch danach zu forschen, ob in Seen mit relativ gefährlichem Ufer die tägliche vertikale Wanderung deutlicher entwickelt sei als anderswo. Freilich wird da vielerlei zu berücksichtigen sein.

Folgende Faktoren vergrößern die Gefährlichkeit des Ufers:

1. Größe und Windreichtum vergrößern die Brandung;

2. Ausgedehnte seichte Partien sind in kaltem Klima gefährlich wegen Durchfrierens, in heißen vielleicht wegen Utberhitzung.

3. Am allergefährlichsten aber ist starker und unregelmäßiger Durchfluß:

a) die Zuflüsse, weil nahrungsarm und untemperiert,

b) der AusfluB als mörderischer Dezimator.

1) Einstweilen muß ich freilich bemerken, daß Ekman in schwedischen Hochgebirge weder vertikale Wanderung noch Uferflucht beobachtet hat. Doch gibt er zu, $\mathrm{da} B$ der Mangel an Booten ganz zuverlässige Untersuchung gehindert habe. (Phyllopoden, Cladoceren und Copepoden der nordschwedischen Hochgebirge. Zool. Jahrb, Syst. Bd. 21. 1904. p. 109., 157 u. a. 
Die zuerst angeführte Ausdehnung der Seefläche aber vergrößert nicht nur die Gefahr, sondern sie verkleinert sie auch, da mit wachsender Seefläche die Möglichkeit dafür, daß Plankton ans Ufer verschlagen wird, sich verringert.

Besonders klein denke ich mir die Gefahr bei Seen ohne oberirdischen Abfluß, die ihrer Kleinheit und geschützten Lage wegen keinen beträchtlichen Wellenschlag haben. Unter solchen Bedingungen könnte man wohl am ehesten Plankton finden, das nicht vertikal wandert.

Wenn ein See unterirdisch abflieBt, könnte die vertikale Wanderung sogar lebensgefährlich werden, und unter solchen Umständen wäre eventuell direkte Wegzüchtung dieser Regulation zu erwarten.

\section{Entstehung des Planktons.}

Meine Hypothese vom Zusammenhang zwischen vertikaler Wanderung und Flucht vor dem Ufer wirft auch ein Licht auf die Entstehung des Süßwasser- und des Meeresplanktons. Da aber der Schuster bei seinem Leisten bleiben soll, will ich mich nur mit dem ersteren beschäftigen. Wesenberg-Lund sagt ${ }^{1}$ ) mit Recht, daB die meisten SüBwasserplanktonten vom Litoral oder vom Boden des SüBwassers stammen; für das Phytoplankton und einen Teil des Zooplanktons möchte ich mit Wesenberg-Lund passive Einwanderung annehmen: Verschleppung vom Litoral her durch Wind und Strömungen, Auftrieb losgelöster Organismen vom Boden her. Für Entomostraken scheint mir aber aktive Einwanderung wahrscheinlicher und zwar aktive Einwanderung unter dem Einfluß negativ heliotaktischer Regulation.

Tatsache ist, dab auch in winzigen Gewässern unter den Verwandten der eulimnetischen Formen negative Heliotaxis verbreitet ist. Daß sie für litorale Formen in ganz anderer Hinsicht als für Plankton zweckmäßig sein kann, ist von V. Bauer für die Mysiden des Neapler Golfes gezeigt worden.

Diese negative Heliotaxis wird nun die Litoraltiere an der Böschung schief abwärts, also vom Ufer weg wandern machen; es wird eine Tendenz vorhanden sein, aus dem Litoral auszuwandern:

1. in mit dem stehenden Gewässer kommunizierende Höhlen,

2. in die Tiefseeregion für solche Formen, die sich nicht von der festen Unterlage emanzipieren können,

3. ins Limneticum für solche, die schon eine genügende Schwebeoder Schwimmfähigkeit besitzen oder sie allmählich erlangen.

Die Lichtflucht können wir also als einen permanenten AnstoB zur Einwanderung ins Limneticum betrachten.

1) Plankton Investigations of the Danish Lakes. General Part. 1908, S. 321-328. 
Stellen wir uns einen bisher planktonlosen See vor mit folgenden vier Vaussetzungen:

1. Starke Konkurrenz um die Nahrung, vielleicht auch um den Sauerstoff am Ufer;

2. Uberfluß an Nahrung (passiv eingewandertem Phytoplankton) und auch an Sauersoff in der limnetischen Region, da hier die Konsumenten fehlen;

3. Am Ufer leben Tiere mit negativer Heliotaxis und wandern unter deren Einfluß allmählich tiefer und in gröBere Uferferne.

4. Unter diesen befinden sich Formen, die schon eine gewisse Schwimmfähigkeit besitzen, also abends von ihren tieferen und uferfernen Standorten zur Oberfläche aufsteigen und die Nahrung sowohl, wie den Sauerstoff am Rande des neuen Phytolimneticums ausnützen können.

So sind die Voraussetzungen zur natürlichen Züchtung gröBerer Schwimm- und Schwebefähigkeit gegeben, wobei sozusagen jeder $\mathrm{cm}$ Fortschritt ins Limneticum Selektionswert hat. Auf diese Art wird sich eine bisher litorale Art in zwei durch Wohnort, Lebensweise und Bau geschiedene Arten spalten (z. B. Sida crystallina in Sida erystallina und Sida limnetica). Nicht durch eine geographische Scheidewand wird hier die neu entstehende Art von der alten isoliert, sondern erstens durch die Gewohnheit, schneller oder weiter vor dem Licht zu fliehen, vor dem Licht und damit vom Ufer weg, also auch weg von der litoralen, weniger stark reagierenden Stammform; zweitens durch die Fähigkeit, bei Lichtabnahme aus der Tiefe vertikal wieder aufzusteigen.

So ist die Lichtflucht vielleicht die primärste Eigentümlichkeit des Zooplanktons, noch um eine Nüance primärer als die Schwebefähigkeit. 